\title{
RESULTS OF THE SIO MASER SURVEY OF THE GALACTIC BULGE IRAS SOURCES
}

\author{
S. DEGUCHI AND N. UKITA \\ Nobeyama Radio Observatory, \\ National Astronomical observatory \\ Minamimaki, Minamisaku, Nagano 384-13, Japan \\ H. IZUMIURA AND T. ONO \\ Department of Astronomy and Earth Sciences, \\ Tokyo Gakugei University \\ 4-1-1 Nukii-kita, Koganei, Tokyo 184, Japan \\ Y. NAKADA \\ Kiso observatory, The University of Tokyo \\ Mitake, Kiso, Nagano 397-13, Japan \\ T. ONAKA AND I. YAMAMURA \\ Department of Astronomy, The University of Tokyo \\ Bunkyo-ku, Tokyo 113, Japan \\ AND \\ O. HASHIMOTO \\ Department of Applied Physics, Seikei University \\ 3-1-1 Kichijouji-kita, Musashino, Tokyo, 180, Japan
}

\section{Introduction}

Evidence for the bar structure in our Galaxy has been shown by Blitz and Spergel (1991b) based on the near-infrared maps of the bulge, by Nakada et al. (1991) based on IRAS point source catalogue, and more clearly by recent COBE maps. However, no clear "dynamical" signature of the bulge bar has been found yet. At optical wavelengths, stellar radial velocities of the bulge stars were observed only at the optical windows and were not observed for the entire region of the bulge because of the dust extinction in this direction. 
The Galactic bulge contains a number of late-type stellar objects which emit maser radiation, for example, the $\mathrm{OH} 1612 \mathrm{MHz}$ maser, and $43 \mathrm{GHz}$ $\mathrm{SiO}$ masers. The galactic disk is tranparent at radio wavelengths. We can survey the bulge stars by $\mathrm{SiO}$ masers and obtain the radial velocities of bulge stars. We can simultaneouly observe two lines of $\mathrm{SiO}$ near $43 \mathrm{GHz}$, i.e., the $\mathrm{J}=1-0$ rotational lines at the first and the second vibrationally excited states. The Nobeyama $45-\mathrm{m}$ radio telescope has a highest sensitivity at 43 $\mathrm{GHz}$. A part of the result of this survey has been published by Nakada et al. (1993), and Izumiura et al. (1994ab). Radio maser surveys by OH have been conducted by several groups. These surveys were mostly devoted to the special small region of the sky, for example, one square degrees near the galactic center with VLA by Lindqvist et el. (1990), or to the entire sky, for example, with Dwingeloo-Effelsberg-Parkes (te Lintel- Hekkert et al. 1990), Arecibo (Lewis et al. 1990), and Nancay (Le Squeren et al. 1992) telescopes. These previous surveys are very extensive and sensitive but not devoted to the bulge stars.

\section{Source selection}

The surveyed region is within 15 degrees from the galactic center and the sources near the galactic plane $\left(|b|<3^{\circ}\right)$ are omitted because there are a lot of galactic disk sources and contamination is severe. Sources are selected by colors, i. e., logarithmic ratio of IRAS 12 and $25 \mu \mathrm{m}$ intensities to be approximately zero. This enables us to pick up the infrared sources with a dust shell of about $T=300 \mathrm{~K}$. Also we impose the IRAS $12 \mu \mathrm{m}$ intensity approximately between 2 and $15 \mathrm{Jy}$. This condition leads us to pick up the infrared sources at the distance of about $8 \mathrm{kpc}$ if the stellar luminosity of the sources is $3 \times 10^{3} L_{\odot}$.

Two-color diagram of the observed IRAS sources indicates that the detected and undetected sources are uniformly distributed in the selected color range. According to the Van der Veen and Habing classification of IRAS stars, these sources belong mainly to the type IIIa, oxygen-rich Mira variables with optically thick dust. Some sources belong to type IIIb, stars with very thick envelope, and to type VIb, the stars thicker at 60 micron. The selected stars have dust shell temperature of about 200-400 K.

\subsection{DISCRIMINATION OF THE DISK SOURCES}

Although we primary select the sources in terms of IRAS colors and intensities, disk sources may contaminate in the samples. To discriminate the disk sources, we further use near infrared photometric data and the periodluminosity relation if available. Whitelock, Feast and Catchpole (1991) measured the period of bulge IR stars in the JHKL bands, especially for stars in 
the galactic latitude between 7 and $8^{\circ}$. The distance to the stars are therefore known from period-luminosity relations for the $|b|=7-8^{\circ}$ strips. For this reason we observed the stars in these strips much more than anywhere else.

For sources in regions other than $|b|=7-8^{\circ}$, we have calculated the probability of a source being the bulge object. The luminosity distribution function of of the bulge IRAS sources can be obtained from the periodluminosity relation given by Whitelock et al. (1991). From this luminosity distribution, we can calculate the probability of the distance to the particular source being within $3 \mathrm{kpc}$ from the galactic center. We have regarded the sources with the bulge probability of more than $50 \%$ as bulge sources and omit the sources with probabilities less than $50 \%$ as disk sources. By this method, we have assigned about $25 \%$ of sources as disk sources.

\section{Observations}

The intensities of the two lines, $\mathrm{SiO} \mathrm{J}=1-0$ rotational lines from the $\mathrm{v}=1$ and 2 states are comparable. In most of sources, two lines of $\mathrm{SiO}$ appear at the same radial velocity. This fact secures detections very much for weak sources in this survey. The maser intensity varies very much. For example, we have observed IRAS 17042-2833 several times. In the first two observations, we could not detect the line. However, at the third observation, two years later from the first observation, we got the clear $\mathrm{SiO}$ signals. We have repeated the observations several times for undetected sources.

\subsection{SIO-OH VELOCITY COMPARISON}

The SiO maser velocity is a good indicator of the stellar velocity (Jewell et al. 1991). About $27 \mathrm{SiO}$ sources have $\mathrm{OH} 1612 \mathrm{MHz}$ masers with the typical double peak profile which is considered to form in the front and receding part of the expanding shell of the star. The center velocity of $\mathrm{OH}$ double peaks has been considered as the stellar velocity. For 27 sources, the $\mathrm{SiO}$ velocity lies within a $2 \mathrm{~km} \mathrm{~s}^{-1}$ from the center velociy of $\mathrm{OH}$. This is a strong indication of the $\mathrm{SiO}$ velocity equals to the stellar velocity.

\subsection{ROTATION OF THE BULGE STELLAR SYSTEM}

The main result of this survey is the plot of radial velocities of $\mathrm{SiO}$ masers with galactic longitude (figure 1). The radial velocity used here is the velocity in the galactic standard of rest, in which the galactic rotation of $220 \mathrm{~km} \mathrm{~s}^{-1}$ of the local standard of rest is subtracted. The best linear fits are shown with the solid line (bulge sources) and dot-dash line (disk sources). The dots deviate from the best fit line appreciably by about $80 \mathrm{~km} \mathrm{~s}^{-1}$ and 


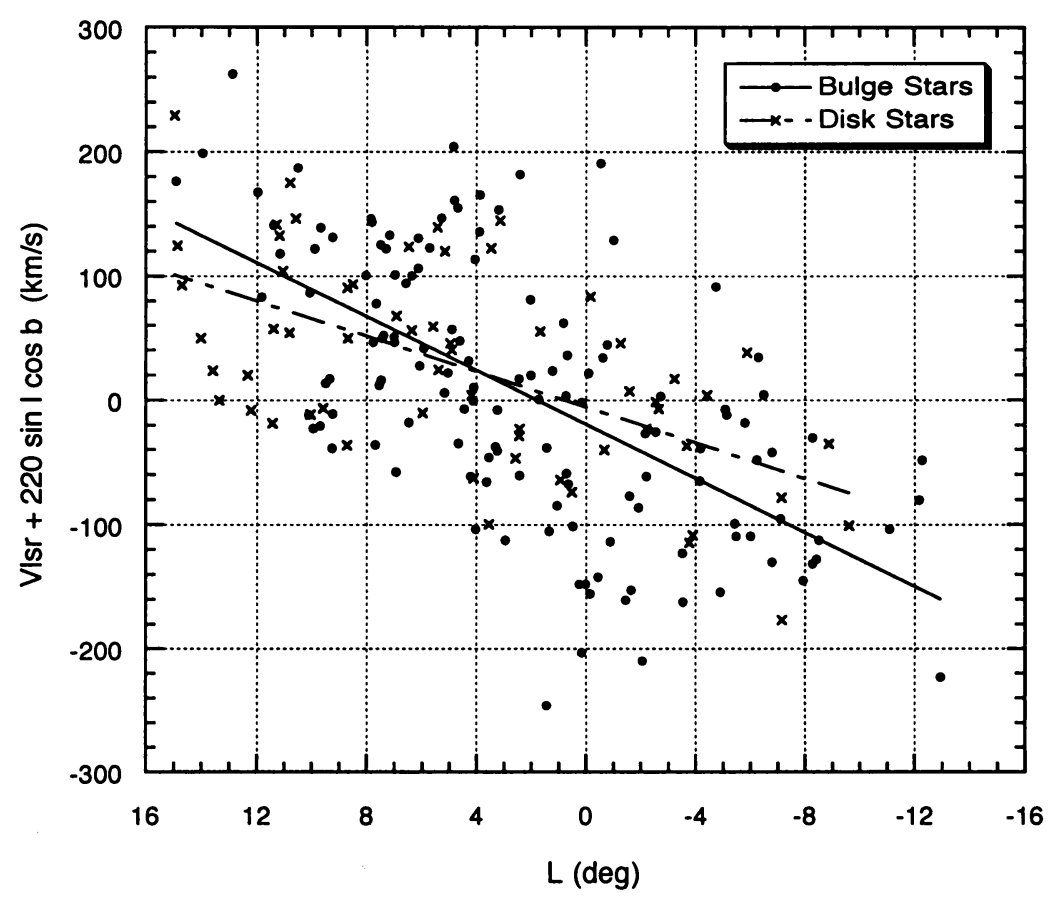

Figure 1. Velocity-Longitude plot for the bulge and disk $\mathrm{SiO}$ maser sources.

this would be a random motion of stars in the bulge. The inclination of the best fit line is about $10 \mathrm{~km} \mathrm{~s}^{-1}$ per degree for bulge sources. This means the galactic bulge stellar system is rotating around the galactic center. A simple computation leads to a rotation period of approximately $9 \times 10^{7} \mathrm{yr}$ and an approximate bulge mass of about $1.4 \times 10^{10} M_{\odot}$ in $2.6 \mathrm{kpc}$.

One peculiarity imediately recognizable in figure 1 is that the radial velocity is shifted by about $20 \mathrm{~km} \mathrm{~s}^{-1}$ from zero at $1=0^{\circ}$. This is quite peculiar because we have a net movement of bulge stars. If we see the best fit to the disk sources, the average shift at the zero longitude is nearly equal to zero. So that the radial velocities of disk stars behave as expected.

\subsection{WHAT CAUSES THE VELOCITY SHIFT ?}

We can consider three explanations for the average overall velocity shift. One is a motion of the local standard of rest, which may be moving toward the galactic center direction. However, this contradicts to the conclusion derived from classic works of HI rotation curve. Kerr (1962) or a recent result of Blitz and Spergel (1991a) concluded that the local standard of rest is moving away from the galactic center with about $14 \mathrm{~km} \mathrm{~s}^{-1}$. Of course, this is based on the measurements of $\mathrm{HI}$ profile, so that it reflects 
the gas motion. Our measurements are based on the stars, so that the gas may have a peculiar motion to the stars.

\subsection{TILT OF THE BULGE}

The other potential explanation of the velocity shift is observational selection. If the bulge is tilted, the radial velocity must be shifted to the negative side for north strip sources and to the positive side for south strip sources. When we observe the bulge from the nothern hemisphere (at Nobeyama), the negative latitude sources may be undersampled. In such a case, the average velocity may be shifted to the negative side.

To check this possibility, we have plotted the north and south bulge sources separately in the Velocity-Longitude diagram. The best fit curves show that the radial velocities for north bulge sources are shifted by about $10 \mathrm{~km} \mathrm{~s}^{-1}$ on average from the radial velocities for the south bulge sources. This indicates that the axis of the bulge stellar system is tilted by about 6 degree from the galactic longitude circle. This result concides with the previous measurement of the tilt of nuclear disk within a observational error, for example, by Burton and Liszt (1978), using HI $21 \mathrm{~cm}$ line. However, we have to stress that taking into account the tilt of the bulge, we still get a net shift, i.e., the average of two rotation curves of north and south bulge sources, of about $19 \mathrm{~km} \mathrm{~s}^{-1}$.

\subsection{POSSIBILITY OF THE BAR STREAMING MOTION}

The other possible cause for the velocity shift is another observational selection effect, that is, the streaming motion of stars in the bar-like potential. When the bulge is elongated, the stars observed from the sun must take an elliptical orbit in Velocity-Longitude diagram. When the ellipticity parameter of the bar varies, the orbit changes. If observers selectively pickup forefront stars, that is, brighter stars, we have more stars with negative radial velocities on average. So that the streaming motion may cause the average velocity shift in the Velocity-Longitude map. Probably this is a signature of the dynamics of the bar-like bulge, unless the local standard of rest is moving toward the Galactic center.

\subsection{PRESENCE OF FOBIDDEN REGIONS}

Another peculiarity to be mentioned is that there are forbidden regions in V-L diagram. There are regions in which no star falls at $(V, l)=(60 \pm$ $\left.35 \mathrm{~km} \mathrm{~s}^{-1}, 3.5 \pm 1^{\circ}\right)$ and $\left(70 \pm 45 \mathrm{~km} \mathrm{~s}^{-1},-3.3 \pm 1.5^{\circ}\right)$. This may be an indicator of streaming motion of the bulge which is caused by the bar-like or elongated structure of the bulge potential. These holes are not so large, 
and not so clear, so that it may be regarded as statistical fluctuations. The probability of the hole being present in the figure is approximately $0.3 \%$ if the star distribution is random.

We have compared the longitude-velocity map of bulge $\mathrm{SiO}$ maser stars with the map of the $21 \mathrm{~cm} \mathrm{HI} \mathrm{line} \mathrm{in} \mathrm{the} \mathrm{nuclear} \mathrm{disk} \mathrm{with} \mathrm{the} \mathrm{same}$ galactic longitude. The HI map was taken from Burton and Liszt (1978). The location of $\mathrm{SiO}$ holes are quite close to the location of the contour dips of HI. HI therefore shows similar dynamical behavior in terms of the forbidden regions. We should note that the HI nuclear disk is on the galactic plane, that is, in $|b|<2^{\circ}$. However, the bulge $\mathrm{SiO}$ maps shown here is the data with $|b|>3^{\circ}$. So that there are no reason to believe that the dynamics of the disk HI gas is similar to the dynamics of $\mathrm{SiO}$ maser stars in the bulge if the gas and stars move independently. Only one explantion for this coincidence is that the bulge is bar-like and the streaming motion of bulge stars influences to the gas motion in the nuclear disk.

\section{Conclusion}

We have observed 313 IRAS sources on the bulge direction and detected $\mathrm{SiO}$ masers in 194 sources. The average rotation velocity is obtained as $-18.7( \pm 7.6)+10.8( \pm 1.2)(l / d e g) k m s^{-1}$. From this rotation curve, we compute the bulge mass within $2.6 \mathrm{kpc}$ as $1.4 \times 10^{10} M_{\odot}$. The bulge rotation axis is considered to be tilted by about $6^{\circ}$. The presence of holes in V-L map may indicate the streaming motion of the stars.

\section{References}

Blitz, L., \& Spergel, D. N. (1991a) ApJ, 370, 205

Blitz, L., \& Spergel, D. N. (1991b) $A p J, 379,631$

Burton, W. B., \& List, H. S. (1978) ApJ, 225, 815

Izumiura, H., Deguchi, S., Hashimoto, O., Nakada, Y., Onaka, T., Ono, T., Ukita, N., \& Yamamura, I. (1994a) $A p J$, (Dec. 10 issue in press)

Izumiura, H., Catchpole, Deguchi, S., Hashimoto, O., Nakada, Y., Onaka, T., Sekiguchi, K., Ono, T., Ukita, N., \& Yamamura, (1994b) ApJ, (submitted)

Jewell, P. R., Sneyder, L. E., Walmsley, C. M., Wilson, T. L., \& Gensheimer, P. D. (1991) $A \& A, 242,211$

Kerr, F. J. (1962) $M N, 123,327$

Le Squeren, A. M., Sivagnam, P., Dennefeld, M., \& David, P. (1992) $A \& A, 254,133$

Lewis, B. M., Eder, J., \& Terzian, Y., (1990) ApJ, 362, 634

Lindqvist, M., Habing, H. J., \& Winnberg, A. (1990) $A \& A, 259,118$

Nakada, Y., Deguchi, S., Hashimoto, O., Izumiura, H., Onaka, T., Sekiguchi, K., \& Yamamura, I. (1991) Nature, 353, 140

Nakada, Y., Onaka, T., Yamamura, I., Deguchi, S., Ukita, N., \& Izumiura, H., (1993) $P A S J, 45,179$

te Lintel-Hekkert, P., Caswell, J. L., Habing, H. J., Haynes, R. F., \& Norris, R. P. (1990) $A \& A S, 90,327$

Whitelock, P., Feast, M., \& Catchpole, R. (1991) $M N$, 248, 276 